\title{
Der erworbene Knick-Plattfuß des Erwachsenen: Eine therapeutische Herausforderung
}

\author{
Markus Wünschel, Nikolaus Wülker
}

\section{Zusammenfassung}

In der Mehrzahl der Fälle ist das abgeflachte Fußlängsgewölbe beim Erwachsenen ohne Krankheitswert. Die Ursache des Knick-Plattfußes ist zumeist in einer insuffizienten Tibialisposterior-Sehne zu suchen. Nur bei Beschwerden sollten therapeutische Maßnahmen ergriffen werden. Die konservative Behandlung hat ihren Stellenwert in den Anfangsstadien der Erkrankung und besteht u.a. aus einer Einlagenversorgung. Im fortgeschrittenen Stadium gibt es eine Vielzahl unterschiedlicher operativer Eingriffe, die miteinander kombiniert werden können, um das abgeflachte Fußlängsgewölbe dauerhaft wieder aufzurichten.
The Acquired Flatfoot of the Adult: A Therapeutic Challenge

The acquired flatfoot of the adult is rarely of clinical significance. The origin of the deformity is mostly an insufficiency of the tibialis posterior tendon. Therapeutic measures should be initiated only if other complaints are present. Conservative treatment including orthotics can only be successful at the initial stage of the disease. A vast selection of operative procedures can be combined to lever up the arch of the flatfoot permanently once the deformity has progressed to an advanced stage.

\section{Einleitung}

Der Knick-Plattfuß des Erwachsenen stellt in der Fußambulanz unserer Klinik einen eher kleinen Patientenanteil dar, obwohl die Fehlstellung in der Bevölkerung relativ häufig anzutreffen ist. Dies liegt daran, dass in der Mehrzahl der Fälle bei - meist milder Deformität - keine Beschwerden vorliegen und auch kein Behandlungsbedarf besteht. Bei den Patienten mit höhergradigen Fehlstellungen und Beschwerden führt eine genaue Anamnese mit körperlicher sowie radiologischer Untersuchung zur Ursache, zumeist der Insuffizienz der Tibialis-posterior-Sehne verschiedener Ausprägungen. Die abgestufte Therapie je nach klinisch-radiologischem Bild wird im Folgenden erläutert.

OP-JOURNAL 2007; 23: $142-147$

(c) Georg Thieme Verlag KG Stuttgart • New York

\section{Ätiologie und Pathogenese}

Die Ätiologie und die Pathogenese des erworbenen Knick-Plattfußes sind bis heute nicht endgültig geklärt. Reihenuntersuchungen von Armeerekruten zeigten, dass der typische, flexible Knick-Plattfuß des Erwachsenen die Funktionsfähigkeit des Fußes nicht beeinträchtigt und lediglich eine Normvariante ohne pathologischen Wert darstellt [7]. Die Fußlängswölbung flacht vom frühen Kindesalter bis zum zwölften Lebensjahr, in dem allmählich die Fußform des Erwachsenen erreicht wird, deutlich ab. Beim Erwachsenen bleibt die Fußform bis etwa zur fünften Lebensdekade unverändert. Danach flacht die Fußwölbung erneut ab.

Durch die valgische Fersenposition beim Knick-Plattfuß kommt es zu einer Abduktion des Chopart-Gelenks, dessen Gelenkebene horizontaler als normal eingestellt wird. Dadurch wird das Chopart-Gelenk entkoppelt und verliert einen Teil seiner Stabilität. Bei abduzier- tem Vorfuß übernimmt die Tibialis-posterior-Sehne die gesamte dynamische Unterstützung der Fußlängswölbung. Bei Anspannung verlagert sie das Navikulare nach medial über den Taluskopf. Dies führt zu einer medial gerichteten Drehung des Talokalkaneargelenks, sodass der Talushals angehoben wird. Der M. tibialis posterior muss beim Gehen das Gewicht des Körpers bei ruhendem Fuß bis zu einem Punkt anheben, an dem das Chopart-Gelenk blockiert ist. Dieser Punkt ist bei allen Füßen gleich, während die Ruhestellung des Fußes von der Höhe der Längswölbung abhängt.

Ist die Längswölbung abgeflacht, muss der Zug des M. tibialis posterior weit kräftiger sein als bei einer normal ausgeprägten Längswölbung. Hier liegt der therapeutische Ansatz zur Korrektur eines Knick-Plattfußes.

Die Tibialis-posterior-Sehne ist die zweitlängste Sehne des Fußes, mit einer Auslenkung von nur ca. $1 \mathrm{~cm}$. Eine Überdehnung, eine Ruptur oder degenerative Veränderungen der Sehne führen zum Knick-Plattfuß (Abb.1). Auch die Folgen von Frakturen, rheumatoider Arthritis oder einer Polyneuropathie können einen Knick-Plattfuß verursachen [3] (Abb. 2a), sind jedoch eher zweitrangig, genauso wie akute traumatische Rupturen der Tibialis-posterior-Sehne [10].

Die Wadenmuskulatur wird durch die Abkippung der Ferse funktionell verkürzt. Die Zugrichtung der Wadenmuskulatur verlagert sich nach lateral und sie bewirkt eine Eversion der Ferse, die die Deformität weiter verstärkt.

Beim flexiblen Knick-Plattfuß besteht bei manchen Patienten gleichzeitig eine Verkürzung der Achillessehne. In diesem Fall ist die Inversion beim Zehenstand eingeschränkt. 


\section{Anamnese und Untersuchungsbefund}

Symptomatische Patienten mit KnickPlattfuß haben zumeist das 5. Lebensjahrzehnt erreicht, sind nicht selten übergewichtig und zeigen oft eine allgemeine Laxizität des Kapsel-Band-Apparates. Frauen sind deutlich in der Mehrzahl betroffen.

Es wird über Wadenkrämpfe, Schmerzen im Verlauf der Tibialis-posteriorSehne medial am Knöchel und eine zunehmende Fußdeformität geklagt. In fortgeschrittenen Fällen kommt es zu einem Impingement der Fibula am Fersenbein mit entsprechenden Beschwerden.

Die klinische Untersuchung zeigt das abgeflachte bis aufgehobene Längsgewölbe häufig kombiniert mit abduziertem Vorfuß. Es fällt eine Schwellung mit Druckschmerz postero-medial am Sprunggelenk auf, teilweise mit Schmerzausstrahlung nach distal. Der Rückfuß steht valgisch, die Fibula kann an das Fersenbein schmerzhaft anstoßen.

Typisch ist das „too many toes sign“: Bei der Inspektion des stehenden Patienten von hinten können vom Untersucher „zu viele“ Kleinzehen erkannt werden, welche normalerweise durch den Unterschenkel und die Ferse verdeckt wären. Dieser Effekt kommt durch die vermehrte Vorfußabduktion zustande (Abb. 2 b).

Ein weiterer häufig anzutreffender Befund ist, dass im Einbeinstand die Ferse aus der valgischen Position nicht in die neutrale bzw. varische Stellung gebracht werden kann (Abb. 3).

Wenn der Fuß bei der klinischen Untersuchung in Neutralstellung gehalten wird und die Dorsalextension im Sprunggelenk auf weniger als $10^{\circ}$ vermindert ist, weist dies auf eine Verkürzung der Achillessehne hin.

\section{Bildgebung}

Die Röntgenuntersuchung beim KnickPlattfuß beinhaltet eine seitliche und dorsoplantare Aufnahme des Fußes im Stehen (Abb. 4). Im Normalfall befindet sich die Talusachse in Verlängerung der Metatarsale-I-Achse. Eine Abkippung der Talusachse um 1-15 Grad nach plantar im Verhältnis zur Metatarsale-IAchse wird als mäßiggradiger Plattfuß

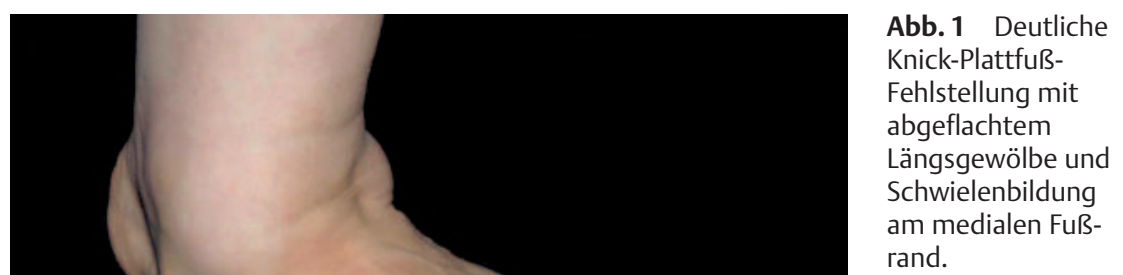

bezeichnet, eine Abweichung um mehr als $15^{\circ}$ als schwerer Plattfuß [1].

Die dorsoplantare Aufnahme dient zur Beurteilung der Vorfußabduktion. Zusätzlich zum nativen Röntgenbild können die Kernspintomografie (Abb.5) oder die Sonografie weitere Informationen zum Zustand der Tibialis-posteriorSehne erbringen, sind jedoch im Normalfall nicht erforderlich.

\section{Therapie}

Die häufigste Ursache für diese Fußdeformität des Erwachsenen ist eine Funktionsstörung der Tibialis-posterior-Sehne (Tab. 1).

Schmerzen stehen im Vergleich zur Fehlstellung im Vordergrund der Beschwerdesymptomatik. Zunächst werden Schmerzen im Sehnenverlauf unter 

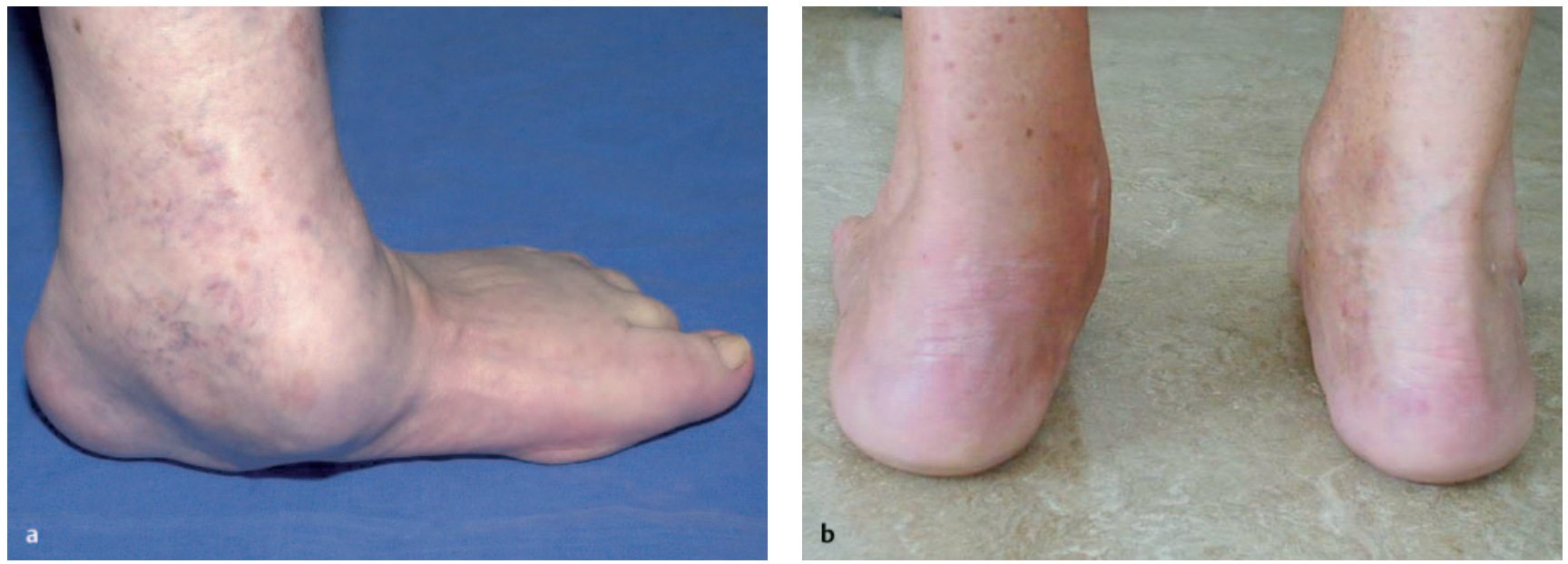

Abb. 2 a und $\mathbf{b}$ a Patient mit rheumatoider Arthritis und imposanter Weichteilschwellung im Verlauf der Tibialis-posterior-Sehne. $\mathbf{b}$ „Too many toes“-Zeichen links.

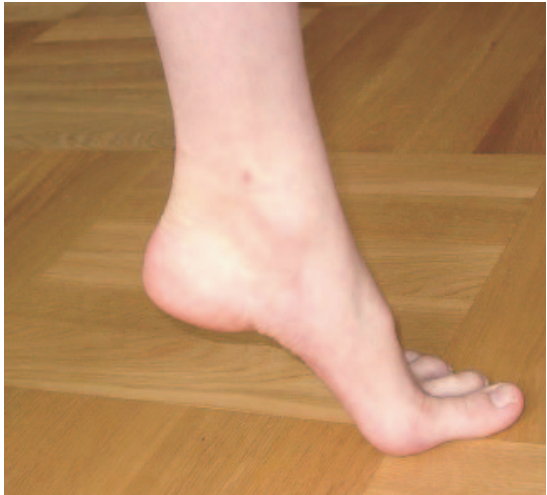

Abb. 3 Das Längsgewölbe kann aktiv nicht mehr vollständig aufgerichtet werden.

dem Innenknöchel angegeben, später im Bereich der Längswölbung und auch lateral im Sinne eines kalkaneofibularen Impingements. Oft handelt es sich um übergewichtige Patienten [2] mit einer seropositiven oder seronegativen entzündlichen Gelenkerkrankung.

Im Anfangsstadium kann eine symptomatische konservative Therapie mit Gewichtsreduktion und Einlagenversorgung versucht werden, die Fehlstellung ist noch flexibel und korrigierbar.

Später entstehen eine kontrakte Valgusdeformität am Rückfuß und eine ebenfalls kontrakte supinatorische Aufdrehung am Vorfuß. Eine kontrakte Deformität wird vorzugsweise mit einer Triple-Arthrodese korrigiert (Abb.6). Die Triple-Arthrodese ist auch bei übergewichtigen Patienten indiziert, die durch eine konservative Behandlung mit Gewichtsreduktion, antiphlogistischer Medikation und orthetischen Maßnahmen

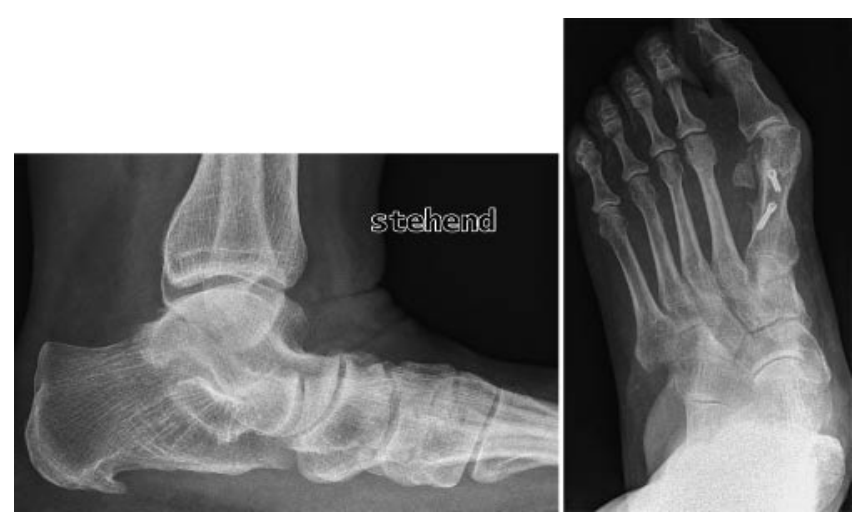

Abb. 4 Die Röntgenaufnahmen zeigen das komplett aufgehobene Längsgewölbe mit Vorfußabduktion.
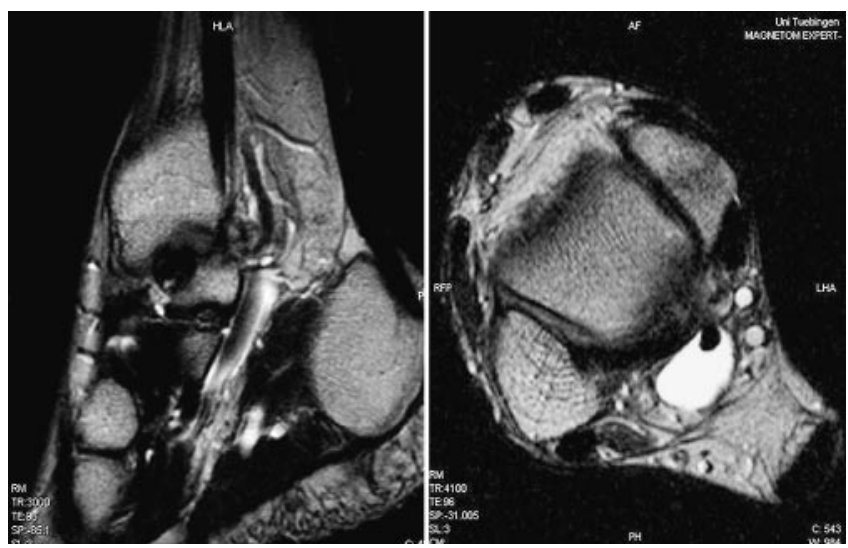

Abb. 5 Kernspintomografischer Nachweis des peritendinösen Ergusses und der Auftreibung im Sehnenverlauf.

keine Besserung ihrer Beschwerden erfahren haben. Bei Patienten mit einer flexiblen Fehlstellung und ohne Übergewicht kann ein Sehnentransfer durchgeführt werden. Dabei bleibt die Beweglichkeit des Fußes erhalten. Für einen Sehnentransfer eignen sich die Flexordigitorum-longus- und die Flexor-hallucis-longus-Sehne, die auf das Navikulare versetzt werden [6]. Ein isolierter Seh- nentransfer bessert zwar in der Regel die Beschwerden, ist jedoch meist nicht in der Lage, die Deformität selbst vollständig zu korrigieren. Dies ist darauf zurückzuführen, dass die genannten Sehnen gegenüber der Tibialis-posterior-Sehne eine wesentlich schwächere Zugwirkung haben. 
Tab. 1 Stadieneinteilung der Insuffizienz der Tibialis-posterior-Sehne [5, 8]

\begin{tabular}{ll} 
Stadium I & - normale Sehnenlänge und -kraft \\
& - milde Symptome \\
& - keine Deformität \\
& - normales Röntgenbild \\
\hline Stadium II & - Sehnenverlängerung und -degeneration \\
& - flexible Deformität \\
& - Schmerzen im Sehnenverlauf \\
& - abgeschwächte Fähigkeit, die Ferse im Zehenstand zu varisieren \\
\hline Stadium III & - Sehnenverlängerung und -degeneration \\
& - kontrakte Deformität \\
& - Schmerz global im Mittelfuß-/Rückfuß \\
& - laterales Impingement \\
& - Unfähigkeit, die Ferse im Zehenstand zu varisieren \\
\hline Stadium IV & - oberes Sprunggelenk mitbetroffen
\end{tabular}

In Kombination mit einer medialen Verschiebeosteotomie des Kalkaneus [8] oder mit einer Verlängerung der lateralen Säule des Fußes durch Distraktionsarthrodese des Kalkaneokuboidgelenks kann die Wirkung des Sehnentransfers verbessert werden.

\section{Operationstechnik}

Beim Erwachsenen werden Knick-Plattfuß-Operationen in Regionalanästhesie oder in Intubationsnarkose und in Blutleere durchgeführt. Die Patienten werden auf dem Rücken gelagert, wobei ein Sandsack unter die gleichseitige Beckenhälfte geschoben wird. Der gesamte Unterschenkel und der Fuß werden desinfiziert und das Bein wird so hoch abgedeckt, dass die Kniescheibe frei bleibt, um die Achsenausrichtung des Fußes besser beurteilen zu können. Das Bewegungsausmaß im oberen Sprunggelenk, im Subtalargelenk und im Chopart-Gelenk wird untersucht. Unter manueller Reposition des Talonavikulargelenks in Neutralposition wird geprüft, ob im oberen Sprunggelenk eine Dorsalextension von mindestens $10^{\circ}$ möglich ist. Wenn dies nicht der Fall ist, muss zusätzlich die Achillessehne verlängert werden. Die Eingriffe an der lateralen Seite des Fußes werden zuerst durchgeführt. Dabei kann es sich um eine Verschiebeosteotomie des Kalkaneus, um eine Verlängerung des Kalkaneus oder um eine Distraktionsarthrodese des Kalkaneokuboidgelenks handeln. Nach Entfernung des Sandsacks unter dem Becken wird an der medialen Fußseite operiert. Über der Tibialis-posterior-Sehne wird ein posteromedialer Hautschnitt angelegt, der hinter dem Innenknöchel beginnt und bis zur Basis des Metatarsale I nach distal zieht. Zahlreiche quer verlaufende Venen werden koaguliert oder unterbunden. Je nach Vorliebe des Operateurs können bei entsprechender Schnittführung sowohl die Fersenbeinverschiebeosteotomie als auch der Sehnentransfer von medial erfolgen (Abb.11).

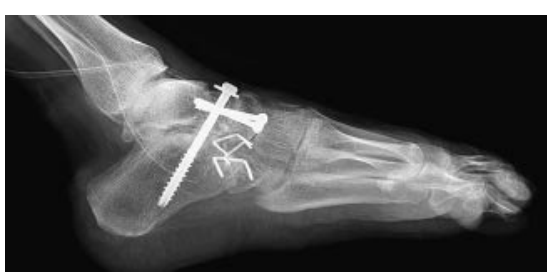

Abb. 6 Postoperatives Röntgenbild nach Triple-Arthrodese.

Zur Knick-Plattfuß-Korrektur des Erwachsenen werden die gleichen Operationsverfahren an der Lateralseite des Fußes wie beim Kind verwendet, insbesondere die Verschiebeosteotomie des Kalkaneus [9] und die Verlängerung der lateralen Säule. Die Verschiebeosteotomie des Kalkaneus wird mit einer Schraubenosteosynthese stabilisiert, nachdem mit einem Kirschner-Draht eine temporäre Fixierung erreicht wurde (Abb. 7). An der Medialseite des Fußes erfolgt in der Regel ein Sehnentransfer.
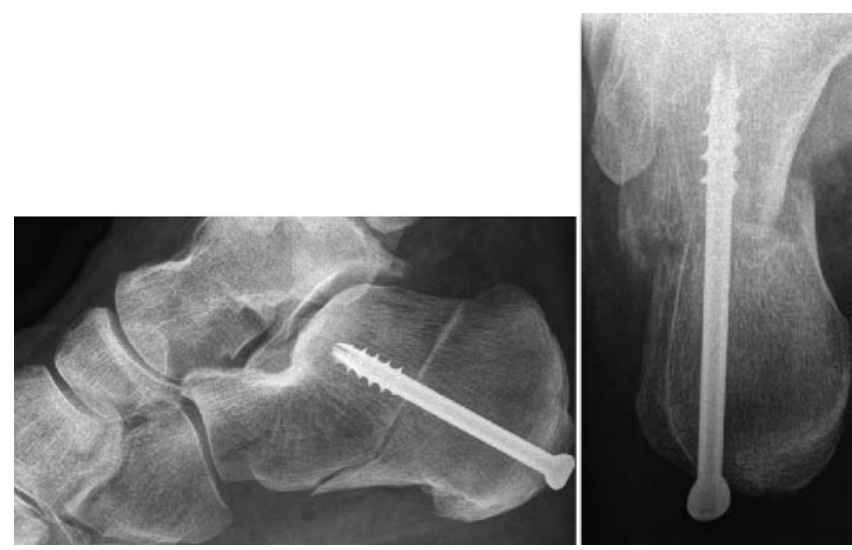

Abb. 7 Operationsergebnis nach medialisierender Fersenbeinosteotomie, die axiale Aufnahme zeigt den korrekten Versatz.

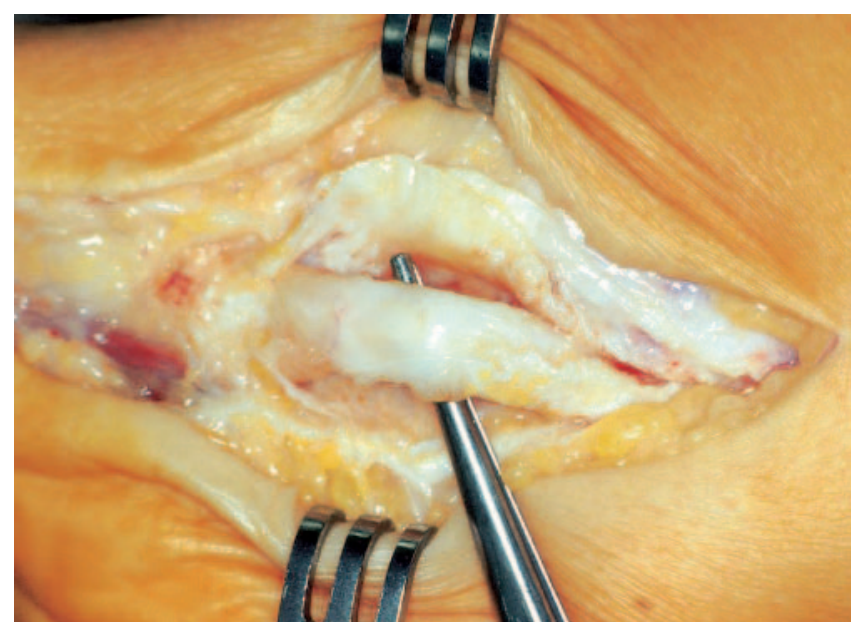

Abb. 8 Verdickte, degenerativ veränderte Tibialis-posterior-Sehne ohne Funktion. 
Die Sehnenscheide des M. tibialis posterior wird eröffnet. Dabei entleert sich häufig etwas seröser Erguss oder es findet sich eine Tenosynovitis (Abb. 8). Die Sehne wird zunächst auf Schwellungen, Degeneration, Vernarbung, Adhäsionen und partielle oder totale Defekte untersucht. Partielle Defekte finden sich regelmäßig auf der Unterseite der Sehne.

Sie werden nach Glättung der degenerativ veränderten Fasern durch intratendinöse Naht versorgt. Komplette Defekte der Tibialis-posterior-Sehne bestehen besonders häufig beim voll ausgebildeten Knick-Plattfuß des Erwachsenen. Die Rupturstelle liegt meist ca. $1 \mathrm{~cm}$ proximal zur Insertion der Sehne am Navikulare, sodass ein kurzer distaler Sehnenstumpf verbleibt.

Durch vorsichtigen Zug am proximalen Sehnenabschnitt lässt sich feststellen, ob Adhäsionen innerhalb der Sehnenscheide entstanden sind. Bei normaler Auslenkbarkeit dieses Sehnenanteils kann die Sehne primär genäht werden, wobei zur Sicherung zusätzlich eine Seit-zu-Seit-Naht an die Flexor digitorum longus-Sehne erfolgen soll [4]. Ist die Tibialis-posterior-Sehne innerhalb ihrer Sehnenscheide verklebt, wird sie hinter dem Innenknöchel durchtrennt.

Die Flexor-digitorum-longus-Sehne wird posterolateral zur Tibialis-posterior-Sehne dargestellt, ihre Sehnenscheide eröffnet und die Sehne bis zur weiter distal gelegenen Sehnenüberkreuzung mit der Flexor-hallucis-longus-Sehne (Henry-Knoten) präpariert (Abb. 9).

Dabei müssen Äste des Venenplexus über der Sehne sorgfältig koaguliert werden, um eine Nachblutung zu verhindern. Die Sehne des M. flexor hallucis longus muss ebenfalls dargestellt und identifiziert werden, um sie nicht versehentlich mit zu durchtrennen. Auch der N. plantaris medialis darf an dieser Stelle nicht verletzt werden. Eine distale Tenodese zwischen der Flexor-digitorum-longus-Sehne und der Flexor-hallucis-longus-Sehne wird nicht routinemäßig empfohlen, da die Sehnen distal zum Henry-Knoten ohnehin funktionell miteinander verbunden sind.

Die Kapsel des Talonavikulargelenks und das Ligamentum calcaneonaviculare werden bei Bedarf gerafft. Etwa $1 \mathrm{~cm}$ lateral zur medialen Kante des $\mathrm{Na}$ vikulare wird an der dorsalen Fläche ein 4,5-mm-Bohrkanal in dorsoplantarer

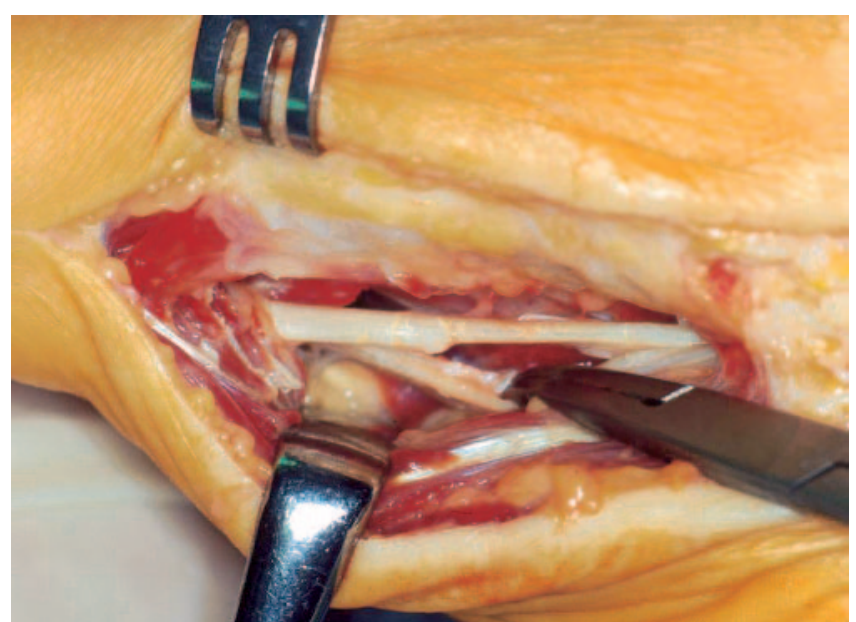

Abb. 9 Am Henry'schen Knoten wird die Flexor-digitorumlongus-Sehne abgesetzt.

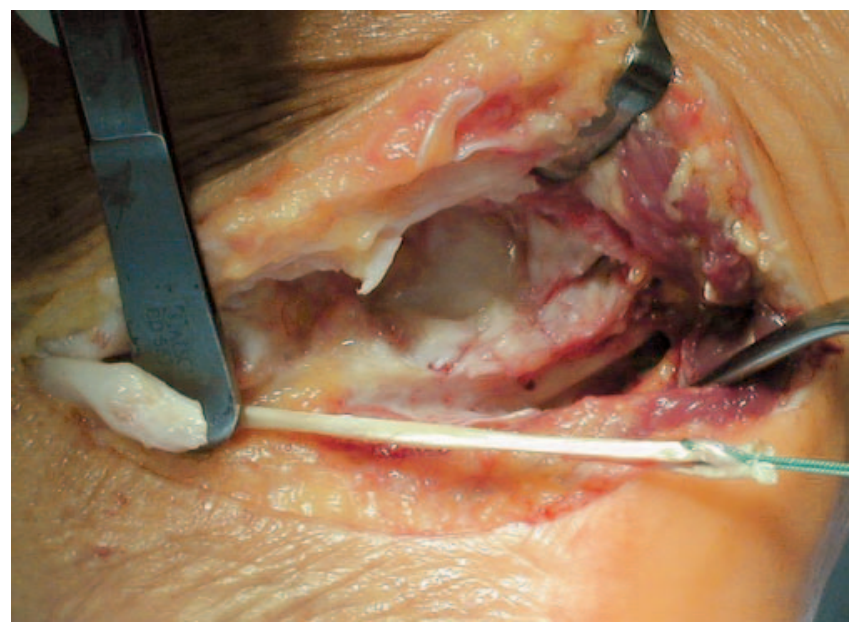

Abb. 10 Armierung der Flex.-dig.-longus-Sehne mit Ethibond, links im Bild der Stumpf der Tib.post.-Sehne.

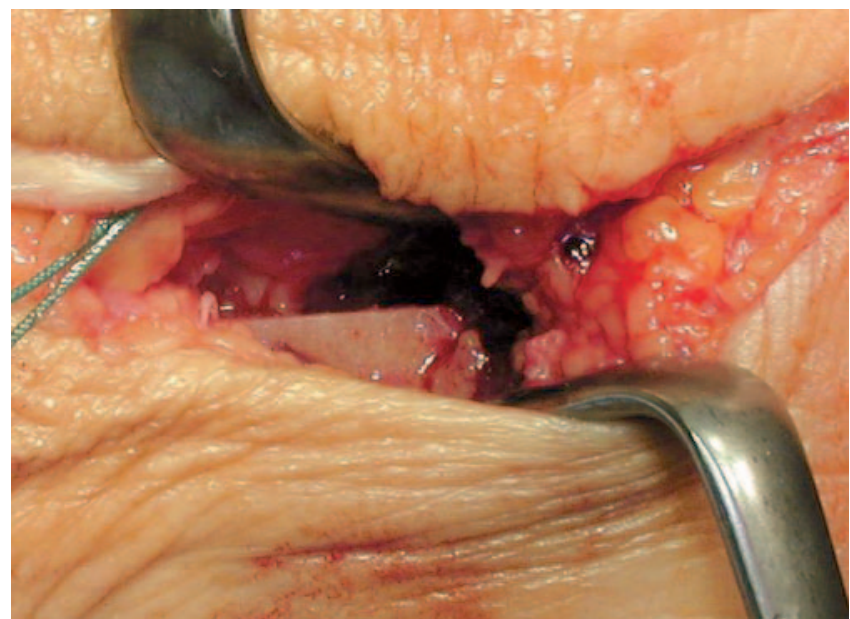

Abb. 11 Versatz des Tuber calcane um ca. $1 \mathrm{~cm}$ nach medial, Zugang von medial.

Richtung angelegt. Die abgelöste Flexordigitorum-longus-Sehne wird von plantar nach dorsal durch diesen Kanal geführt. Dazu wird das Sehnenende mit einem Faden im Sinne einer Kessler-Naht armiert (Abb.10). Das Fadenende kann auch mit einer Drainage durch den Kno- chenkanal angesaugt und die Sehne durchgezogen werden, die umgebenden Weichteile müssen dafür entsprechend präpariert sein. Die Sehne muss ausreichend vorgespannt werden. Dazu wird sie in die Mittelposition zwischen der Ruhestellung der Sehne und ihrer maxi- 
malen Auslenkung gebracht und unter maximaler Inversion und leichter Plantarflexion des Fußes fixiert. Auch möglich ist die Fixierung der submaximal ausgelenkten Sehne unter Neutralstellung des Fußes.

Die Sehne wird am Periost des Navikulare oder transossär nach Anfertigung eines kleinen Bohrloches befestigt. Je nach Sehnenlänge kann sie um das Navikulare nach plantar geführt und mit sich selbst vernäht werden. Die Festigkeit der Sehnenfixation lässt sich überprüfen, indem der Fuß im oberen Sprunggelenk maximal dorsalextendiert und plantarflektiert wird. Wenn die zuvor durchtrennte Tibialis-posterior-Sehne proximal noch eine gute Auslenkung aufweist, wird der proximale Sehnenstumpf Seit-zu-Seit an der Flexor-digitorum-longus-Sehne befestigt, um eine zusätzliche Krafteinleitung zu ermöglichen [4].

Der Wundverschluss erfolgt schichtweise nach Einlegen einer Drainage. Der Fuß sollte in Neutralstellung in einem Gips immobilisiert werden. Nach 48 Stunden wird der erste Verband gewechselt und die Drainage entfernt. Nach Abschwellen der Weichteile wird ein geschlossener Unterschenkelcast angelegt, der bis 6 Wochen postoperativ verbleibt. Nach dieser Zeit wird je nach Ergebnis der Röntgenkontrolle die Belastung über weitere 6 Wochen zunehmend gesteigert. Insgesamt 10 Wochen nach der Operation können erste Übungen gegen Widerstand zur Kräftigung der Inversion durchgeführt werden. Vorsichtige Übungen zum Erreichen des beidseitigen Zehenstandes sind mit Abstützung der Längswölbung möglich.

\section{Komplikationen}

Intraoperative Komplikationen können entstehen, wenn wichtige Strukturen nicht ausreichend dargestellt werden. Beim Spalten der Sehnenscheide des M. flexor digitorum longus ist das GefäßNerven-Bündel im Tarsaltunnel gefährdet. Deshalb empfiehlt es sich, die Sehnenscheide durch die posterolaterale Wand der Tibialis-posterior-Sehnenscheide zu eröffnen. Bei Durchtrennung der Flexor-digitorum-longus-Sehne am Henry-Knoten können die benachbarten Strukturen verletzt werden, weshalb die Sehne nur unter direkter Sicht abgelöst werden darf. Die Sehne wird von plantar nach dorsal durchtrennt, um die plantar zur Sehne liegenden Strukturen, insbesondere die Flexor-hallucis-longusSehne und den N. plantaris medialis, nicht zu verletzen.

Postoperative Komplikationen wie Hämatome, ein Kompartmentsyndrom oder protrahierte Schwellungen können auftreten. Durch sorgfältige Blutstillung, durch Einlegen einer Drainage und durch Anlegen eines gut gepolsterten postoperativen Kompressionsverbands können diese Komplikationen weitgehend vermieden werden. Ein Rezidiv der Fehlstellung wird durch eine Arthrodese der entsprechenden Gelenke korrigiert. Bei einer kontrakten Deformität erfolgt eine Triple-Arthrodese.

\section{Schlussfolgerung}

Die richtige Indikationsstellung für den Zeitpunkt der operativen Therapie kombiniert mit der zielgerichteten Auswahl und Kombination der unterschiedlichen operativen Verfahren sind der Schlüssel zum Erfolg der operativen Therapie des erworbenen Knick-Plattfußes im Erwachsenenalter.

\section{Literatur}

1 Bordelon RL. Hypermobile flatfoot in children. Comprehension, evaluation, and treatment. Clin Orthop Relat Res 1983; 181: 7 - 14

2 Fuhrmann RA, Trommer T, Venbrocks RA. The acquired buckling-flatfoot. A foot deformity due to obesity? Orthopäde 2005; 34: $682-689$

${ }^{3}$ Holmes GB Jr. Mann RA. Possible epidemiological factors associated with rupture of the posterior tibial tendon. Foot Ankle 1992; 13: $70-79$

4 Jahss MH. Spontaneous rupture of the tibialis posterior tendon: clinical findings, tenographic studies, and a new technique of repair. Foot Ankle 1982; 3: $158-166$

5 Johnson KA, Strom DE. Tibialis posterior tendon dysfunction. Clin Orthop Relat Res 1989; 239: $196-206$

${ }^{6}$ Mann RA. Rupture of the tibialis posterior tendon. Instr Course Lect 1984; 33: 302-309

7 Milgrom C, Giladi M, Simkin A, Stein M, Kashtan H, Margulies J, Steinberg R, Aharonson $Z$. The normal range of subtalar inversion and eversion in young males as measured by three different techniques. Foot Ankle 1985; 6: $143-145$

${ }^{8}$ Myerson MS. Adult aquired flatfood deformity: treatment of dysfunction of the posterior tibial tendon insufficiency. J Bone Joint Surg [Am] 1996; 78: 780-792

9 Trnka HJ, Easley ME, Myerson MS. The role of calcaneal osteotomies for correction of adult flatfoot. Clin Orthop Relat Res 1999; 365: $50-64$

10 Zwipp H, Dahlen C, Amlang M, Rammelt S. Injuries of the tibialis posterior tendon: diagnosis and therapy. Orthopäde 2000; 29: $251-259$

\section{Dr. med. Markus Wünschel \\ Oberarzt}

Prof. Dr. med. Nikolaus Wülker

Ärztlicher Direktor

Orthopädische Klinik der

Eberhard-Karls-Universität Tübingen

Hoppe-Seyler-Straße 3

72076 Tübingen 